\title{
Erratum zu: Sozialpolitik im Finanzföderalismus - Hartz IV als Antwort auf die Krise der Kommunalfinanzen
}

\author{
Anke Hassel · Christof Schiller
}

Erratum zu: Polit Vierteljahresschr (2010) 51:95-117

DOI 10.1007/s11615-010-0006-Z

Die Online-HTML-Version des Beitrages wurde leider ohne den zweiten Autor veröffentlicht.

Die Autoren-Zeile muss lauten: Anke Hassel · Christof Schiller

Im Adressenblock fehlt: Dipl.-Verw.wiss. C. Schiller, E-Mail: schiller@hertie-school.org

Online publiziert: 22.05 .2010

(C) VS-Verlag 2010

Die Online-Version des Original-Artikels ist zu finden unter doi: 10.1007/s11615-010-0006-z

Prof. Dr. A. Hassel $(\bowtie) \cdot$ Dipl.-Verw.wiss. C. Schiller Hertie School of Governance, Friedrichstr. 180, 10117 Berlin, Deutschland

E-Mail: hassel@hertie-school.org 